BULLETIN Bulletin hispanique

HISPANIQUE Université Michel de Montaigne Bordeaux

112-1| 2010

Actes du Colloque « langue, littérature, littéralité »

\title{
Le signifiant et son double
}

\section{Raphaël Estève}

\section{OpenEdition}

Journals

Édition électronique

URL : http://journals.openedition.org/bulletinhispanique/1139

DOI : 10.4000/bulletinhispanique.1139

ISSN : 1775-3821

\section{Éditeur}

Presses universitaires de Bordeaux

\section{Édition imprimée}

Date de publication : 1 juin 2010

Pagination : 275-286

ISBN : 978-2-86781-692-5

ISSN : 0007-4640

Référence électronique

Raphaël Estève, "Le signifiant et son double », Bulletin hispanique [En ligne], 112-1 | 2010, mis en ligne le 01 juin 2013, consulté le 01 mai 2019. URL : http://journals.openedition.org/ bulletinhispanique/1139; DOI : 10.4000/bulletinhispanique.1139 


\title{
Le signifiant et son double
}

\author{
RAPHAËL EstÈve \\ Université Michel de Montaigne Bordeaux
}

Le propos de cet article est d'opérer un rapprochement entre d'une part une théorie philosophique monomaniaque, celle de Clément Rosset dont "la philosophie du Réel " postule que nous ne cessons de surimposer à ce dernier un "double ", et d'autre part la théorie et la pratique de l'analyse littérale.

El presente artículo ofrece un acercamiento entre, por un lado, una teoría filosófica monomaniaca, la de Clément Rosset, cuya "filosofía de lo real" propugna que no hacemos más que sobreimprimirle un doble a la realidad, y, por otro, la teoría y la práctica del análisis literal.

This article intends to bring together, on the one hand, a mono-maniac philosophical theory, Clément Rosset's, whose "Philosophy of Real" postulates that we stop superimposing a 'double' to reality, and on the other hand, theory and praxis of literal analysis.

Mots-clés : Littéralité - Nadine Ly - Clément Rosset - Cervantès - Philosophie du Réel.

LA LeCTURE, il y a quelques temps, d'un petit texte de Clément Rosset
intitulé "L'Espagne des apparences " ${ }^{1}$ ' 'ai immédiatement su que j'avais
trouvé le biais par lequel je pourrais un jour me joindre, à ma façon et à mon

1. Clément Rosset, «L'Espagne des apparences », in Le choix des mots, Paris, Minuit, 1995.

$B H i$, Tome 112 , nº 1 - juin 2010 - p. 275 à 286. 
échelle, à l'hommage qu'il me semblait important de rendre à Nadine Ly. Ce que je ne savais évidemment pas, c'est que l'occasion me serait donnée de le faire dans un cadre aussi propice et entouré de collègues aussi éminents.

Mon intention est ici d'opérer un rapprochement entre d'une part une doctrine philosophique monomaniaque, la philosophie du Réel de Clément Rosset, et d'autre part la théorie et la pratique de l'analyse littérale.

Il serait en fait plus exact de dire que je vais simplement accompagner ce rapprochement qui me semble à bien des égards préexister, ne serait-ce que parce que Rosset, dans le texte que j'ai mentionné, illustre son propos doctrinaire en s'appuyant sur deux œuvres de Cervantès, Le Retable des merveilles et plus sommairement $\mathrm{La}$ Cave de Salamanque. Je proposerai à mon tour, une fois énoncé le principe de la philosophie de Clément Rosset, un développement littéraire de son commentaire du premier de ces deux textes.

Dès l'entame de l'article que Frédéric Bravo, dans le recueil Limage dans le tapis, consacre à l'analyse littérale, on peut lire qu'un des termes employés par Nadine Ly pour la définir est celui de " déontologie ${ }^{2}$ ». Je souhaite précisément présenter ici l'analyse littérale comme une dé-ontologie, c'est-àdire comme une défiance vis-à-vis de l'ontologie classique.

La source à laquelle puise cette dernière, le poème de Parménide, évoque on le sait deux voies : la voie de l'Être, nécessaire, unique, et immuable, et celle de la doxa, autrement dit de l'opinion, caractérisée à l'opposé de la première. C'est ainsi que les commentateurs de ce poème " au moins depuis Platon $^{3}$ ", sont parvenus pour reprendre les termes de Clément Rosset à l'idée d'un être "ontologique». Ontologique, à savoir "dégagé de tout rapport avec l'existence commune, c'est-à-dire avec la réalité sensible, temporelle et changeante ${ }^{4}$. On sait ce qu'il advient : la voie de l'opinion rend du fait de son inconstance toute connaissance impossible, et le monde sensible nous interdit donc l'accès à toute forme de vérité autre qu'accidentelle. Dans la philosophie platonicienne, la vérité est à l'inverse présentée comme relevant d'un ordre dit intelligible précisément parce sa validité transcende les accidents du sensible. Cette validité générale ${ }^{5}$ est généralement ressentie un compromis établi par Platon. Chez Parménide, l'Être est un. Cela équivaut

2. Frédéric Bravo, "L'analyse littérale ", in Littéralité III, L’image dans le tapis, Bordeaux, Collection de la Maison des Pays Ibériques, 1997, p. 43.

3. Clément Rosset, Principes de sagesse et de folie, Paris, Minuit, 1991, p. 14.

4. Ibid., p. 14.

5. Héritée de la géométrie pythagoricienne. 
à dire "qu'il n'y a qu'une seule "manière" d'être " ". Dès lors, toute chose qui " est " est de la même manière que toute autre. Or force est de constater que les êtres existants sont différents les uns des autres. Platon doit donc parvenir à penser ce qui selon l'interprétation qu'il fait de Parménide relève du nonêtre, c'est-à-dire cette multiplicité ${ }^{7}$. Il va l'accomplir en dissociant plusieurs niveaux de réalité. Platon ramène le multiple du sensible à un nombre limité de " genres » ou de "formes ». Et l'on sait que ce qui caractérise l'ontologie ${ }^{8}$ de Platon c'est bien le fait que "la "Forme" (ou "Idée") soit "plus réelle que ce dont elle est la Forme ${ }^{9}$ ". L'intelligible qui seul permet d'accéder au vrai est ainsi décrété plus réel que le sensible. C'est là le réalisme de Platon, son interprétation hiérarchisante du poème de Parménide.

Clément Rosset souhaite entendre ce poème d'une tout autre manière pour à son tour y fonder sa philosophie. Sa volonté s'exprime dans l'interprétation qu'il livre de la sentence du sixième fragment du poème de Parménide, et plus exactement de la fin de son premier vers : " esti gar einai ", traduisible par "l'Être est ", " il y a de l'Être », ou, comme le fait Rosset, " ce qui est, est ". Le verbe " einai " exprimant à la fois en grec "l'idée d'être et d'exister ${ }^{10}$ ", Rosset ne voit aucune raison pour privilégier le premier sens au détriment du premier. Pour lui au contraire seul ce qui existe est. C'est en ce principe que va résider son affirmation du Réel. Et la seule définition qu'il en livrera est une définition négative qui prend le contrepied de l'ontologie platonicienne. Selon cette dernière, «le réel immédiat n'est admis et compris que pour autant qu'il peut être considéré comme l'expression d'un autre réel, qui seul lui confère son sens et sa réalité ${ }^{11}$ ». L'objet matériel et sensible est donc déprécié au profit de ce dernier. Et le Réel immédiat et concret que ces objets constituent en est paradoxalement réduit à n'être que le double, le mauvais double, de cet autre.

Toute la philosophie de Rosset consiste à la fois à illustrer - en puisant notamment dans la littérature - et à regretter ce déni de l'effectivité du réel qui nous fait le ramener sans cesse à un autre que lui. Il y aurait chez nous une incapacité congénitale à accepter l'identité de la chose à elle-même, le dénuement tautologique de $\mathrm{A}=\mathrm{A}$ que Rosset veut lire chez Parménide.

6. Francis Wolff, L'Être, le maître, le disciple, Paris, Quadrige/Presses Universitaires de France, 2000, p. 23.

7. C'est là son " parricide».

8. Une ontologie de la forme que l'on opposera à l'ontologie aristotélicienne de l'individu.

9. Francis Wolff, L'Être, le mâtrtre, le disciple, p. 32.

10. Clément Rosset, Principes de sagesse et de folie, op. cit., p. 9.

11. Clément Rosset, Le réel et son double, in L'école du réel, Paris, Minuit, 2008, p. 38. 
C'est à mon sens un sentiment similaire qui incite Nadine Ly dans son article de 1986 consacré à l'analyse littérale des textes à appeler de ses vœux la prise en compte d'un même "définitivement détaché et libéré de l'Autre ${ }^{12}$ ".

Et on entendra pareillement, à l'échelle des constituants de ces textes, le refus d'une appréhension hypostasiée - je reprends ici la formulation de Frédéric Bravo - du signifiant. Hypostasier le signifiant, c'est en faire l'émanation d'un autre que lui, qu'il ne contribuerait qu'à représenter. En réduisant le signifiant à n'être que le véhicule du signifié, autrement dit, en le subordonnant à cet autre, on néglige son identité à lui-même, sa matière ${ }^{13}$.

Il me semble que tout cela revient à poser que la Réalité du texte ou du signifiant, l'existence la plus immédiatement sensible qu'ils proposent, c'est la leur. Pour l'analyse littérale, le signifiant est avant tout égal à lui-même. Je ramènerai donc pour le moment le respect de la Lettre à l'acceptation, par devers les « résistances » de cette dernière soulignées par tous, au dénuement tautologique $\mathrm{A}=\mathrm{A}$.

Si je m'autorise ce rapprochement entre l'analyse littérale et la philosophie de Clément Rosset, c'est à l'instigation d'un texte que ce dernier consacre à ce qu'il présente bel et bien, malgré toutes les précautions d'usage, comme le " tempérament espagnol ». Un tempérament en très forte adéquation avec la vision du monde, autrement dit du Réel, de l'auteur.

Rosset, coutumier des illustrations littéraires, convoque ici pour étayer son propos deux ouvres de Cervantès, Le Retable des merveilles et La cave de Salamanque. Il s'attarde davantage sur la première que sur la seconde, et j'accentuerai pour ma part ce déséquilibre en ne traitant pas ici de cette dernière.

L'argument de cette dernière est le suivant : un charlatan nommé Chanfalla arrive dans un village avec son acolyte Chirinos et parvient à persuader les notables du lieu d'assister à la représentation de son théâtre ambulant, le Retable des merveilles, dont la particularité est selon les dires de Chanfalla de n'être perceptible qu'aux espagnols de pure souche. Le temps de la représentation venu, le charlatan la fait immédiatement précéder de l'annonce du spectacle qui va se dérouler sous les yeux de l'assistance. On le sait, il ne se passe rien, aucun spectacle n'est représenté, mais les spectateurs, pour garantir aux yeux des autres la pureté de leur lignage, vont, à une

12. Nadine Ly, «La littéralité ", in Littéralité I, Bordeaux, Collection de la Maison des Pays Ibériques, 1989, p. 194 ("La littéralité c'est le même du texte, c'est le texte propre ").

13. Plotin, figure de proue du néoplatonisme répugnait ainsi à être représenté par un artiste : son corps n'était qu'un reflet, la représentation de ce corps serait ainsi le reflet d'un reflet. 
exception près, faire comme si le spectacle avait lieu, et en jouer eux-mêmes la comédie. Ils la jouent si bien qu'ils finiront par prendre tout aussi à la légère l'arrivée imminente dans le village d'un régiment de soldats.

Avant de livrer l'interprétation que Clément Rosset propose de cette œuvre de Cervantès, je voudrais mettre ici à profit le fait que j'ai passé plusieurs jours à rechercher la référence du texte du philosophe.

Je m'explique. Mon souvenir de l'argument du Retable était d'autant plus net qu'il préexistait à la lecture du texte recherché. L'intention doctrinaire de Rosset, toujours la même, ne faisait elle non plus aucun doute pour moi. En revanche, la logique du raisonnement reliant les deux, l'œuvre et l'intention, demeurait beaucoup plus incertaine tant que ce texte n'avait d'existence que dans ma mémoire.

Je me demandais si le propos de Rosset était de postuler la distanciation de Cervantès par rapport à ce que je concevais inadéquatement comme l'illusion dont étaient victimes ses personnages. Mais cela ne me semblait pas satisfaisant. La défaillance de ma mémoire me ramenait en réalité au travers que Rosset entend perpétuellement dénoncer. Je ne faisais pas que chercher la référence bibliographique de ce texte, je cherchais également, commettant peut-être ce "pêché de réalité " pointé par Frédéric Bravo reprenant l'expression de Jean-Claude Chevalier, à sauver la référence linguistique de son objet cervantin. Il est vrai que dans le texte dont je parle, finalement retrouvé puisqu'il s'intitule "L'Espagne des apparences ", dernier chapitre de l'ouvrage Le choix des mots, la relation établie par Rosset entre l'argument cervantin et sa philosophie du Réel demande à mon sens à être explicitée. C'est ce à quoi je vais m’employer, pour expier ma volonté d'avoir voulu «sauver la référence ", et plus sérieusement pour expliquer ce que j'entends par là.

Je commence, comme il se doit, par reproduire le commentaire que propose le philosophe. Rosset écrit qu'il n'est personne dans l'assistance du Retable des merveilles pour s'étonner que la chose annoncée ne soit pas suivie d'effet visible ou tangible. Car ce ne serait là qu'un effet "superfétatoire au gré du tempérament espagnol, persuadé que la chose annoncée consiste en sa propre annonce et en elle seule ${ }^{14}$ ". Je laisse pour le moment de côté le tempérament espagnol pour mieux insister sur la difficulté à faire coïncider dans mon souvenir l'argument du récit de Cervantès avec le credo de Rosset, le fait, il le répète dans ce même texte, qu' "il n'y a pas à chercher d'être audelà de l'apparence dont [la chose] s'affuble ${ }^{15}$ ". Car le coup de force est là,

14. Clément Rosset, «L'Espagne des apparences », op. cit., p. 143.

15. Ibid., p. 143. 
quand on nous dit que cette apparence n'est garantie ici que par la parole du charlatan alors même qu'aucune image, "aucune "apparence", au sens strict d'impression visuelle ne la soutient ${ }^{16}$ ». Ce qu'il fallait comprendre, c'est que l'apparence, le Réel matériel, c'est la parole en tant qu'elle est proférée, et donc qu'elle vient à l'existence. Dans un texte, c'est même la première de toutes les existences. Clément Rosset parle donc d'une apparence " réduite à sa plus simple et plus pure expression, d'une chose qui n'existe que par le mot qui la suggère ${ }^{17}$ ». Et c'est ce qui lui permet d'affirmer que Chanfalla emporte l'adhésion d'un public convaincu « qu'il n'y a pas de différence sensible entre être et être suggéré ${ }^{18}$ ".

Je vais tenter de compléter d'une façon plus littéraire la démonstration de Rosset en rapportant des propositions énoncées en d'autres lieux de son œuvre à la lettre du texte de Cervantès.

Il me semble tout d'abord que la non-représentation associée au retable, le fait qu'en dehors des mots prononcés par Chanfalla, il ne se passe rien, affranchit réflexivement le texte tout entier de sa propre fonction de représentation. Affirmer de la sorte qu'il n'y a rien en dehors des mots de ce texte équivaut à rendre non avenu ce à quoi ces mots seraient susceptibles de référer y compris en son sein. Exemples parfaits de signifiants sans référent, les mots de Chanfalla, annonçant ce que les spectateurs vont voir, ne sont plus des reflets ni des doubles. Ils ne représentent plus, et ils ramènent de ce fait notre attention sur leur propre existence. En ce sens, ils se font advenir eux-mêmes à l'existence. Et il n'est donc pas anodin que le charlatan, étymologiquement parleur, commence par s'adresser performativement à l'auteur du Retable qu'il " conjure " de montrer ses " merveilleuses merveilles ». La performativité, on le sait, substitue à la représentation un évènement en soi, une réalité qu'institue le mot. Ce principe performatif va contaminer la description par Chanfalla de ce que les spectateurs doivent voir. Dire que cette description ne décrit rien c'est précisément commettre notre péché de réalité ou de référence et négliger la dimension prétéritionnelle des textes : dire ce que l'on voit et dire ce que l'on ne voit pas revient exactement au même dès que l'on postule la seule réalité du langage. Les mots existent matériellement de la même façon dans les deux cas, indépendamment de leur modalisation. Ils sont, une fois signifiée leur émancipation référentielle, infalsifiables, comme les verbes performatifs impropres à être pensés en termes de vérité et de fausseté.

16. Ibid., p. 143.

17. Ibid., p. 143.

18. Ibid., p. 144. 
Tout ce que prétendent voir les spectateurs du Retable existe donc bel et bien textuellement. Ils ne demandent d'ailleurs qu'à prendre Chanfalla au mot, si on se fie à ce que l'alcade assure au greffier : « haced vos que me hablen a derechas, que yo entenderé a pie llano ${ }^{19}$."

On peut difficilement se situer davantage au pied de la lettre. Et ce n'est donc pas un hasard que l'Alcalde soit précisément le premier à voir, ou mieux : à dire qu'il voit.

Car les spectateurs déclarent eux-mêmes le spectacle que de ce fait ils instituent à leur tour. Et on serait bien mal inspiré de conférer moins de réalité à leurs mots qu'à un hypothétique Réel du texte échappant à l'empire de ces derniers. Il faudrait pour cela, en fermant les yeux sur le fait que ces personnages sont eux-mêmes irréels, stratifier les niveaux de réalité. Or le texte n'est que surface, tout y est matériellement sur le même plan : «a pie llano».

Si je souhaitais prendre à son tour au mot Clément Rosset, je dirais que la parole de Chanfalla manifestement réduite par le texte de Cervantès à elle-même et à rien de plus, rien d'autre, constitue un cas extrême de parole oraculaire. Il s'agit là du premier paradigme proposé par Rosset pour expliquer notre tendance à la duplication du Réel. Dans les mythes impliquant une instance oraculaire, l'accomplissement de la prédiction survient y compris lorsque l'intéressé a tout mis en œuvre pour l'éviter. Dans tous les cas, cet accomplissement le surprend, il s'attendait toujours à autre chose. Il a " jusqu'à la fin, a misé sur la grâce d'un double ${ }^{20}$ ". Or ce qui doit arriver arrive et la plupart du temps dans les termes exacts décrits par l'oracle : c'est précisément cette identité qui surprend le prédestiné.

Prendre au mot Rosset ce serait donc ici dire que la parole de Chanfalla est oraculaire au sens où ce qui advient est exactement ce qui est dit, et rien de plus que les mots qui le disent. Dans le mécanisme de l'illusion oraculaire, je noterai également que cette coïncidence à soi, cette simplicité limpide du réel, " apparait comme l'absurdité majeure aux yeux de l'illusionnééci ". Elle est une absurdité car le réel n'est rien d'autre que lui-même.

Il est dès lors tentant de mettre à profit le nom du créateur supposé du Retable, puisque ce dernier s'appelle, selon Chanfalla, Tontonelo. Ce nom déclare une bêtise mettant en relation étroite la crédulité affichée par les spectateurs du Retable et l'idiotie du Réel qui vient d'être évoquée.

19. Miguel de Cervantes Saavedra, El retablo de las maravillas in Teatro, Paris, Editorial Bouret, 1947, p. 249 (je souligne "pie llano").

20. Clément Rosset, Le réel et son double, op. cit., p. 36.

21. Ibid., p. 36. 
Certains phénomènes textuels renvoient en effet au thème de l'identité à soi.

Le signifiant Tontonelo affiche ainsi une duplication interne de la syllabe " ton ». Cela me semblerait insuffisant pour le décréter égal à lui-même si la mise en contexte de ce nom ne semblait pas le faire tendre vers une certaine forme de tautologie. Une tautologie que la sonorité du signifiant Tontonelo m'a d'ailleurs selon toute vraisemblance également inspirée. La première chose qui est en effet prédiquée 22 à propos de Tontonelo, c'est qu'il est de Tontonela. Dans sa réponse aux explications que lui demande le gouverneur sur le nom du Retable juste avant l'apparition textuelle de Tontonelo, Chanfalla déclare que s'il s'appelle Retable des merveilles, c'est à cause du caractère merveilleux des choses que l'on y montre. Et on se rappelle que plus tard, lorsque le même Chanfalla invoque au moment de la représentation l'auteur du Retable, il somme Tontonelo de montrer ses "maravillosas maravillas $^{23}$ ". Et il le somme de le faire sur le champ ("incontinente ») c'està-dire dans une immédiateté que j'entends comme la coöncidence avec luimême du moment de son énonciation. Cette inscription tautologique du signifiant Tontonelo dans le texte déclare donc figurativement l'identité à soi de tous les autres signifiants, celle-là même qui fera que le spectacle du Retable ne sera rien d'autre que les mots qui le disent.

Il faut bien entendu reconnaître que cette dimension figurative réintroduit une forme de représentation : si c'est de la stricte identité à soi que l'on parle, si $A$ se réduit à $A$, alors on ne peut rien en dire, puisque qu'on ne peut le ramener à rien d'autre qu'à lui-même. Or prédiquer, faire advenir le sens, c'est ramener de l'autre au même.

En théorie pour Rosset, le Réel n'a pas de sens car lui imprimer un sens serait l'envisager comme un ensemble clos et donc le transcender. C'est pourquoi il ne définit jamais théoriquement ce Réel, car il n'est identique qu'à lui-même. C'est là son idiotie, il n'entre pas en relation : il est incausé et demeure à jamais insolite, car il n'a pas de double.

L'analyse littérale cherche au contraire à mettre un sens à jour. Elle se veut une intelligence du texte vis-à-vis duquel elle entend d'ailleurs produire ellemême un discours ${ }^{24}$.

La littéralité ne renonce donc pas à toute forme de transcendance, puisqu'elle postule un établissement systémique de relations causales appelées

22. Par Chirinos.

23. Miguel de Cervantes Saavedra, El retablo de las maravillas, op. cit., p. 251.

24. Et elle se démarque de la pathologie pointée par les psychiâtres (qui voient le patient éconduit essayer de prendre littéralement la porte), précisément parce que l'objet de son attention est textuel, et que le Réel et la matière du texte, ce sont les mots. 
" motivations ». Systémique, c'est-à-dire interne au monde clos constitué par le texte. On pourrait dire que la littéralité stratifie l'unicité du signifiant qui demeure malgré tout un de ses postulats. Ainsi, au-dessus du signifiant, on aurait son inscription dans un système qu'il contribue, il est vrai, à édifier ; en dessous de lui les strates, oubliées ou pas, de son histoire propre. Le signifiant entendu littéralement n'est donc pas exactement tautologique, sans quoi, encore une fois, on ne pourrait rien en dire. C'est le point de divergence avec la théorie du Réel de Rosset. Avec sa théorie, mais pas avec l'expression pratique de cette théorie. On voit mal sinon comment le philosophe pourrait, même négativement, définir le réel sans tomber dans une sorte de contradiction performative.

Or, en dépit de sa conviction théorique réitérée, Rosset prédique à propos du Réel, comme Chirinos prédique dans le texte de Cervantès, au sujet de Tontonelo, sans pour autant invalider l'inscription tautologique de ce dernier. Si je le mentionne, c'est que l'auteur du Retable est lui aussi appréhendé par le biais de son apparence, cette barbe hypertrophiée qui le qualifie aux yeux de cet Alcalde si littéral de "sage ». Je ne me répéterai pas: je stipulerai seulement que cette qualification de sagesse sur la foi du mot qui la nomme est plus réelle que l'hypothétique véracité diégétique qui la contredirait ; cette apparence et les mots du personnage qui la commente sont sa seule réalité.

D'ailleurs, l'Alcalde qui entend au pied de la lettre est celui-là même qui étend l'empire de Tontonelo au-delà du Retable. Il attribue au même auteur l'arrivée pourtant apparemment effective des trente hommes d'armes. On croit que c'est là sa crédulité. Mais ne cherche-t-on pas en croyant cela encore une fois à sauver la référence ? Car qu'est-ce qui différencie textuellement les deux événements ? Dès lors que Le Retable est une représentation sans objet diégétique, il contamine de son irréalité référentielle la description de l'arrivée des soldats : il nous rappelle que l'objet de la représentation de cette dernière est un objet fictif. Et c'est littéralement l'analogie que l'Alcalde déclare en faisant de Tontonelo l'auteur des soldats. Ces deux représentations n'ont pas la même instance, mais elles ont au final, à la surface plane du texte la même existence matérielle, littérale. Une existence de mots égaux à eux-mêmes.

De ce fait, la sagesse déclarée par cet Alcalde, lui-même projection de Tontonelo et de sa bêtise, n'est peut être pas si inepte que ça. Peut-être que s'exprime au travers de Tontonelo le fin mot du Réel : s'accommoder de l'idiotie de ce Réel est en tout cas, à n'en pas douter, la plus haute sagesse à laquelle la philosophie de Rosset peut aspirer. 
Reste à présent à faire un sort à ce " tempérament espagnol ».

C'est bien ici encore une sagesse, un sophos, que Rosset veut nous signifier quand il déclare que le peuple espagnol peut " être tenu pour à la fois le plus philosophe et le moins métaphysicien qui soit ${ }^{25}$ ". La relation de cause à effet est patente : c'est parce qu'il n'est pas métaphysicien, autrement dit, parce qu'il est rétif à l'ontologie classique que l'Espagnol est philosophe : il n'attend rien de plus du Réel que lui-même. Et on mesure l'investissement affectif que cela représente pour Rosset, espagnol d'origine, quand il déclare que toute chose existante est perçue en Espagne, comme " tragique ". Tragique, puisque " réduite à son état apparent ${ }^{26}$ ". Tragique enfin comme La philosophie tragique ${ }^{27}$, titre du premier ouvrage de Clément Rosset. Si je fais écho à cette anthropologie nationale, toujours forcément suspecte, et davantage encore chez un philosophe de la singularité, c'est parce que les remarques par lesquelles l'auteur la déclare m'ont semblé particulièrement légitimer la pertinence de l'approche littérale dans le cas de l'espagnol. Rosset écrit en effet que la sensibilité espagnole est « rebelle à l'enseignement de saint Paul, recommandant aux Corinthiens dans une célèbre Epître de privilégier l'esprit au détriment de la lettre ${ }^{28} »$. Aux oreilles d'un Espagnol, qui «ne tient pour réel que ce qui s'en manifeste ${ }^{29}$ ", et considère par conséquent l'esprit comme indiscernable de la lettre qui le suggère " toucher à la lettre, c'est toucher à la chose même ${ }^{30} "$.

Rosset n'étant pas à un paradoxe près, il fait de la dissociation unique parmi les langues européennes dérivées du grec et du latin, de l'être entre " ser " et " estar " un contre-argument à sa thèse. Cette différence nous dit-il « est au fond complètement étrangère au tempérament espagnol ${ }^{31}$ ". Pour lui, en effet, ce tempérament est au contraire incapable de distinguer véritablement entre être par essence et être par accident.

On pourrait parfaitement accréditer la thèse inverse et dire que l'existence d' " estar " témoigne au contraire de la prise en compte pleine et déterminée d'une dimension de l'être dont l'existence est concrète et localisée : en espagnol, cette dimension de l'être n'est pas absorbée ni indéterminée par un signifiant global.

25. Clément Rosset, "L'Espagne des apparences », op. cit., p. 150.

26. Ibid., p. 154.

27. Clément Rosset, La philosophie tragique, Paris, PUF, 1960.

28. Ibid., p. 155.

29. Ibid., p. 155.

30. Ibid., p. 156.

31. Ibid., p. 151. 
De fait, si j'avais moi-même dû choisir dans la langue espagnole un contreexemple aussi peu invalidant que celui de Rosset, je me serais intéressé à l'expression de l'existence impersonnelle. Ce n'est pas ici le lieu de mener cette étude en détail, mais je formulerai tout de même quelques remarques qui me mèneront à ma conclusion.

On l'a vu, la philosophie du Réel s'oppose à l'interprétation que l'ontologie classique a livrée du Poème de Parménide.

Celle-ci culmine avec Heidegger. L'unicité qui caractérise l'Être chez Parménide peut sembler impliquer qu'il y a une manière d'être commune à tout ce qui est, et donc, qu'en amont de ce qui existe, il y a un principe que Heidegger, lecteur de Parménide, appelle l'Être. Or ce que la philosophie heideggérienne déplore, ce dont elle se lamente, c'est l'oubli de cet Être.

$\mathrm{Si}$ j'en parle ici c'est qu'on a toujours recours, pour faire comprendre ce qu'est l'Être heideggérien au même biais linguistique. Celui de la quantification existentielle : dans "il y a ", ou dans " hay ", ce "y " semble permettre l'expression d'un principe plus indéterminé encore que l'impersonnel.

Or il est amusant de constater que ce que la langue, elle, a oublié dans sa diachronie, c'est précisément le contraire de l'Être : c'est l'existant concret. En effet, ce " $y$ » qu'on entend à présent comme un non lieu absolu ${ }^{32}$, c'est bien du «IBI » latin qu'il procède. Cet anaphorique adverbial déclare un lieu tout à fait effectif. La langue espagnole a donc oublié en agglomérant ce "y " qu'il exprimait une localisation concrète de l'existence. Désormais, $y$ devient le signifiant abstrait, avec le quantificateur hay, de l'existence au temps fondamental du présent, " [...] un lieu d'espace privilégié et fondamental : celui même où s'inscrit le présent temporel [...], et qui n'est autre que le présent spatial de l'existence $[\ldots] »^{33}$.

On voit que la langue par ce type d'oubli semble accréditer la thèse de Rosset : ce qu'on risque d'oublier c'est plutôt l'existant que l'Être. Que le quantificateur " hay " déclare l'existence fondamentale au prix de l'amnésie de ce $Y$, c'est même ce qu'on enseigne aux étudiants d'espagnol en linguistique diachronique, une linguistique qui lorsqu'elle dresse la généalogie des composantes du signifiant, nourrit les remotivations étymologiques de l'analyse littérale.

32. L'expression de la transcendance, l'ex-sistence, "le locatif absolu, sans lieu " (Henri Maldiney, Penser l'homme et la folie, Grenoble, Million, 1991, p. 222).

33. Maurice Molho, "Essai sur la sémiologie des verbes d'existence en espagnol ", in Linguistiques et langage, Bordeaux, éd. Ducros, 1969, p. 57-99. Voir aussi Marie-France Delport, Deux verbes espagnols $=$ Haber et Tener, Paris, Éditions Hispaniques, 2004, notamment le chapitre V, « L'emploi unipersonnel », p. 473-475. 
Le Y. Vous me voyez venir, et vous voyez bien car je vais effectivement terminer sur une note de fantaisie onomastique.

Clément Rosset décline son thème de l'identité à soi en posant le problème de l'identité personnelle. Continuateur en cela de Hume, Rosset affirme qu'il n'y a d'identité que sociale et officielle. Nous décrétons souvent la facticité de notre identité sociale au profit d'une supposée identité authentique au sens platonicien du terme, qui selon Rosset est l'illustration parfaite du fantasme duplicateur.

Lors d'une conversation où elle échangeait avec Nadine Ly à propos de leurs itinéraires respectifs, une collègue forte de son prénom puisqu'elle s'appelle Ana (qui se trouve être également le prénom - qu'on se gardera donc scrupuleusement de déclarer plus «vrai " - de Nadine Ly) s'est exclamée avec le naturel et la bienveillance qui la caractérisent :

Mais finalement, vous n'êtes pas du tout "Nadine Ly".

Je ne sais pas si Ana est platonicienne, et je me crois au moins autant qu'elle sous l'emprise des illusions que dénonce Clément Rosset. Mais je reste pourtant persuadé que Nadine Ly est très exactement Nadine Ly, Nadine Ly avec un Y : elle lit ce qui est là. 